\title{
The OptoGait Motion Analysis System for Clinical Assessment of 2D Spatio-Temporal Gait Parameters in Young Adults: A Reliability and Repeatability Observational Study
}

\author{
Julia Carbajales-Lopez ${ }^{1}$, Ricardo Becerro-de-Bengoa-Vallejo ${ }^{2}{ }^{\circledR}$, Marta Elena Losa-Iglesias ${ }^{1}{ }^{(\mathbb{C}}$, \\ Israel Casado-Hernández ${ }^{1}$, Maria Benito-De Pedro ${ }^{1}$, David Rodríguez-Sanz ${ }^{2, *}$, \\ César Calvo-Lobo ${ }^{2}$ (I) and Marta San Antolín ${ }^{3}$ \\ 1 Faculty of Health Sciences, Universidad Rey Juan Carlos, 28922 Alcorcon, Spain; \\ Podoclinicacarbajales@gmail.com (J.C.-L.); marta.losa@urjc.es (M.E.L.-I.); israelcasado@yahoo.es (I.C.-H.); \\ mariabenito1309@gmail.com (M.B.-D.P.) \\ 2 School of Nursing, Physiotherapy and Podiatry, Universidad Complutense de Madrid, 28040 Madrid, Spain; \\ ribebeva@ucm.es (R.B.-d.-B.-V.); cescalvo@ucm.es (C.C.-L.) \\ 3 Department of Psychology, Universidad Europea de Madrid, Villaviciosa de Odón, 28670 Madrid, Spain; \\ marta.sanantolin@gmail.com \\ * Correspondence: davidrodriguezsanz@ucm.es
}

Received: 12 May 2020; Accepted: 22 May 2020; Published: 28 May 2020

check for updates

\begin{abstract}
Background: Walking clinical assessments are commonly used to check for possible gait modifications in healthy subjects, in patients with trauma or neurological conditions, or after a surgical procedure. Spatio-temporal walking variables are studied in depth to achieve a complete evaluation. The purpose of this study was to perform an observational study of the reliability and repeatability of the OptoGait portable photoelectric cell system. Methods: An observational study of the reliability and repeatability of the OptoGait portable photoelectric cell system was carried out. Results: Walking point, walking point gap, step width, and walking base variables, which are deeply related to gait and posture, have an almost perfect reliability. Conclusion: The OptoGait motion analysis device is a reliable system for clinical assessment of static and dynamic foot pressures. Clinicians should feel confident adding this analysis system to their daily professional tools where repeatable measures of gait measurements are important for diagnosis and treatment selection, and also compare efficacy or testing differences between different pathologic conditions. Further, these obtained data can be formed as a baseline for future studies, and to check a right sample size for new research that use the OptoGait motion analysis system.
\end{abstract}

Keywords: gait; reliability; analysis; foot

\section{Introduction}

Walking clinical assessments are commonly used to check for possible gait modifications in healthy subjects, in patients with trauma or neurological conditions, or after a surgical procedure. Spatio-temporal walking variables have been studied in depth to achieve a complete evaluation [1-6]. Several biomechanical methods are commonly used to check gait, such as the orthopedic test and recording with a video camera. In the last decade, the number of computerized analyses has increased in order to obtain highly reliable measurements $[7,8]$.

Many new tools have been generated that can perform gait analysis. Different gait assessment methods as a force platform provide a static analysis about foot plantar pressures, but OptoGait 
provides a complete dynamic analysis of foot movements and space-temporal relationships. One such tool, the OptoGait Cell-System (OptoGait, It), allows clinicians to quantify spatio-temporal parameters with a photoelectric cell-based analysis system. The software provides clinicians with 19 variables about gait conditions, so this device offers deep and acceptable information about the movement of subjects. OptoGait motion analysis could provide an added value for clinical assessment of gait and posture in different clinical disciplines. The reliability of the OptoGait device has been tested by different research studies $[2,3,9,10]$. The main purpose of this study was to check OptoGait's inter-session and intra-session reliability for both feet in young adults.

\section{Methods}

From April 2017 to March 2018, we developed observational research of the reliability and repeatability of the OptoGait portable photoelectric cell system. The ethics and research committee of the University Rey Juan Carlos (Madrid, Spain) approved this observational study with internal code number 0202201702917. Informed consent was previously obtained from all the subjects.

\subsection{Sample Size}

The sample size was obtained using the Fisterra software from the Epidemiologic and Biostatistical unit of Coruña University. It was obtained on the basis of the minimal number to detect a Pearson coefficient (0.4) with a confidence interval of $95 \%$ and an $\alpha$-value of 0.05 in a two-tailed test with a desired power of $80 \%$.

A sample of 87 healthy participants ( 44 men, 43 women) was selected. Subjects were excluded for the following reasons: cardiovascular, neurological, or musculoskeletal problems, or foot pain (previous 6 months). Participants with prior lower limb surgery, spine surgery, or any condition that may have affected their gait and/or posture were also excluded.

Four gait parameters were measured with the OptoGait system: step width, walking base, walking points, and walking point gap. The definitions of the studied parameters are as follows: step width is the distance between the midpoint for the right and left feet. Walking base is the distance between the medial point of the right and left feet. Walking points are the midpoints between both feet (their conjunction defines the line of progression; it is expressed with positive values if there is a deviation to the left and with negative values if it is to the right). Walking point gap is the progressive variation of the current walking point from the previous one.

The system was checked and calibrated by the manufacturer. Subjects walked between two parallel bars (with ninety-six LED diodes on each bar). While participants walk in the system, their feet block the photoelectric signal between the two parallel bars, allowing the system to obtain step width, walking base, walking points, and walking point gap data. The OptoGait system was connected to a personal computer with OptoGait software. The data were obtained at a sampling frequency of $1000 \mathrm{~Hz}$.

Before the data acquisition trials, the subjects developed one single previous trial. The first OptoGait bar-sensor was placed $40 \mathrm{~cm}$ from the subject's position in order to successfully record the first step. All the recruited subjects were barefoot for the training trial and data acquisition trials. Participants were asked to walk in a normal way over a 10-m walkway at a pace that was adequate for them to walk normally, starting with their dominant foot [11]. Subjects started with both feet at the same line in front of the OptoGait corridor, and when the rater confirmed an adequate position, they started walking and finished after crossing the other bar; the start and end points were placed at a 2-m distance in order to reduce the effects of acceleration/deceleration. The subjects completed this recording protocol 8 times/session and the average-values were tested for intra-session reliability. For inter-session reliability, different measures were obtained seven days after the first assessment. Only complete steps in the sensor-defined areas were accepted in the computer analysis. Other authors such as Besser show that analysis from 6 to 8 strides were adequate to achieve reliable and right values (defined as $95 \%$ CI) [12]. All measurements were recorded by the same experienced rater. 


\subsection{Statistical Analysis}

The Kolmogorov-Smirnov test was carried out to assess the normality of the data, and the data were considered normally distributed if $p>0.05$. The quantitative variables were reported as mean SD with a $95 \%$ CI. To test for any differences in the obtained data between subjects, an independent $t$-test was carried out. In order to check any differences in gait data between different sessions paired t-tests were carried out.

For the intra-session and inter-session trials, the intra-class correlation coefficient (ICC) $[1,3]$ was Determined to evaluate the test-retest reliability of the variables. For the inter-session analysis, the average data of the 8 measurements was used. We carried out our experiments using this order of agreement [13]: 0.20 or less, slight agreement; $0.21-0.40$, fair; $0.41-0.60$, moderate; $0.61-0.80$, substantial; and 0.81 or greater, almost perfect. Based on previous research [14], we used clinical evaluations with reliability coefficients over 0.90 to increase the probability of measurement reliability.

Coefficients of variation (CVs) were analyzed for absolute comparison of the different data. The CVs were obtained to test the intra-session reliability. The CV was obtained as the mean normalized to the SD. A higher CV value shows that the values are more heterogeneous. The analysis was carried out with values from right and left feet. The minimal detectable change (MCD) is defined as a valid change in score that is not due to chance. The repeatability coefficient (CR) of the value below which the absolute difference between two repeated test results may be expected to lie with a probability of $95 \%$. The concordance limit is defined as the limits of agreement and are the confidence limits of this difference.

We calculated the standard errors of the mean (SEM) in order to test the range of error of each gait obtained data that we analyzed. The SEM value was obtained between the different sessions from the ICCs and SDs.

At the end, values of normality $(\mathrm{VN})$ of the subjects were defined for the studied data with the system. They were calculated from the following equation: $\mathrm{VN}=$ Mean $+/ 1.96 * \mathrm{SD}$. A $p$ lower than 0.05, with a known 95\% CI, was considered statistically significant (SPSS for Windows, version 24.0; SPSS Inc., Chicago, IL, USA).

\subsection{Results}

Descriptive demographic data are shown in Table 1. Studied variables were non-parametrically distributed. The analysis of intra-session reliability values for the initial session are shown in Table 2 . The average walking point value was $3.933 \pm 1.371$ with a mean ICC of $0.885(0.82-0.925)$ and a SEM value of 0.465 . The highest ICC value was obtained for the step width variable (mean ICC of 0.908 (0.872-0.936) and a SEM of 0.196) and the lowest ICC value was obtained for walking point gap (mean ICC of $0.758(0.670-0.829)$ and a SEM of 0.091). For all the studied variables, the ICC achieved strong values (i.e., almost perfect). Table 2 shows the $\mathrm{VN}$ for all the variables.

Table 1. Descriptive demographic values for total sample.

\begin{tabular}{ccc}
\hline & Total $(n=87)$ & CI \\
Mean SD $(95 \%$ CI $)$ & \\
\hline Age (Years) & $27.5 \pm 1.79(27.1-27.9)$ & \\
Weight $($ Kg $)$ & $64.9 \pm 9.9(62.8-66.9)$ & \\
Height $(\mathrm{cm})$ & $168.5 \pm 5.2(167.4-169.6)$ & $95 \%$ \\
BMI & $22.7 \pm 2.66(22.2-23.3)$ & \\
Foot Size (UE Size) & $39.8 \pm 2.29(39.4-40.3)$ & \\
\hline
\end{tabular}

Abbreviations: BMI: body mass index; CI: confidence interval; SD: standard deviation. 
Table 2. Analysis of intra-session reliability for both feet in first session.

\begin{tabular}{cccccccc}
\hline Gait Variables & Mean (SD) & CV (\%) & ICC (95\% CI) & SEM & \%ERROR SEM & MDC & Values of Normality \\
\hline Walking Points & $3.93 \pm 1.37$ & 34.8 & $0.88(0.82-0.92)$ & 0.46 & 11.81 & 1.28 & $3.93 \pm 2.68(1.24-6.61)$ \\
Walking Point & $1.96 \pm 0.18$ & 9.40 & $0.75(0.67-0.82)$ & 0.09 & 4.62 & 0.25 & $1.96 \pm 0.36(1.60-2.32)$ \\
Gap & $11.0 \pm 0.64$ & 5.86 & $0.90(0.87-0.93)$ & 0.19 & 1.77 & 0.54 & $11.0 \pm 1.26(9.75-12.2)$ \\
Step width & 13.7 & $0.81(0.74-0.86)$ & 0.18 & 5.94 & 0.51 & $3.14 \pm 0.84(2.29-3.98)$ \\
Walking base & $3.14 \pm 0.43$ &
\end{tabular}

Abbreviations: SD: standard deviation; CV: coefficients of variation; ICC: intraclass correlation coefficient; CI: confidence interval; SEM: standard error of the mean; MDC: minimal detectable change.

The analysis of the intra-session reliability values for the second trial is shown in Table 3 . The mean walking point value was $4.075 \pm 1.201$ with a mean ICC of $0.861(0.803-0.904)$ and a SEM value of 0.448 . The highest ICC value was again obtained for step width (mean ICC of $0.903(0.868-0.932)$ with a SEM of 0.170 ) and the lowest ICC value was for walking point gap (mean ICC of $0.668(0.547-0.765)$ and a SEM of 0.118). For all the studied variables, the ICC achieved strong values (i.e., almost perfect). The VN for the walking points, walking point gap, step width, and walking base variables are shown in Table 3.

The analysis of the inter-session reliability data is presented in Table 4 . The highest ICC value was obtained for walking points (mean ICC of $0.943(0.913-0.963)$ with a SEM of 0.024) and the lowest ICC value was obtained again for walking point gap variable (mean ICC of $0.863(0.790-0.910)$ and a SEM of 0.003 ). The $\mathrm{VN}$ for the walking points, walking point gap, step width and walking base variables for the inter-session reliability data are shown in Table 4.

Table 3. Analysis of intra-session reliability for both feet in second session.

\begin{tabular}{cccccccc}
\hline Gait Variables & Mean (SD) & CV (\%) & ICC (95\% CI) & SEM & $\begin{array}{c}\text { \%ERROR } \\
\text { SEM }\end{array}$ & MDC & Values of Normality \\
\hline Walking Points & $4.07 \pm 1.20$ & 29.4 & $0.86(0.80-0.90)$ & 0.44 & 11.9 & 1.24 & $4.07 \pm 2.35(1.72-6.43)$ \\
Walking Point Gap & $1.97 \pm 0.20$ & 10.3 & $0.66(0.54-0.76)$ & 0.11 & 5.98 & 0.32 & $1.97 \pm 0.40(1.57-2.38)$ \\
Step width & $11.2 \pm 0.54$ & 4.86 & $0.90(0.86-0.93)$ & 0.17 & 1.51 & 0.54 & $11.2 \pm 1.07(10.1-12.3)$ \\
Walking base & $3.27 \pm 0.45$ & 13.7 & $0.85(0.79-0.89)$ & 0.17 & 5.29 & 0.51 & $3.27 \pm 0.88(2.38-4.15)$ \\
\hline
\end{tabular}

Abbreviations: SD: standard deviation; CV: coefficients of variation; ICC: intraclass correlation coefficient; CI: confidence interval; SEM: standard error of the mean; MDC: minimal detectable change.

Table 4. Analysis of inter-session reliability for both feet.

\begin{tabular}{|c|c|c|c|c|c|c|}
\hline & $\begin{array}{l}\text { Mean First } \\
\text { Session } \pm \text { SD } \\
(\text { CI 95\%) }\end{array}$ & $\begin{array}{l}\text { Mean Second } \\
\text { Session } \pm \text { SD } \\
\quad(C I 95 \%)\end{array}$ & $\begin{array}{c}\text { INTER-SESSION } \\
\text { Mean } \pm \text { SD } \\
(\text { CI } 95 \%)\end{array}$ & $p$ Value & ICC $(95 \%$ CI $)$ & CV (\%) \\
\hline Walking Points & $3.93 \pm 1.37$ & $4.07 \pm 1.20$ & $4.00 \pm 0.10$ & 0.37 & $0.94(0.91-0.96)$ & 2.51 \\
\hline Step width & $11.0 \pm 0.64$ & $11.2 \pm 0.54$ & $11.1 \pm 0.14$ & 0.06 & $0.90(0.87-0.93)$ & 1.33 \\
\hline \multirow[t]{2}{*}{ Walking base } & $3.14 \pm 0.43$ & $3.27 \pm 0.45$ & $3.20 \pm 0.09$ & 0.37 & $0.93(0.89-0.95)$ & 2031.7 \\
\hline & SEM & $\begin{array}{l}\text { \%ERROR } \\
\text { SEM }\end{array}$ & MDC & CR & $\begin{array}{c}\text { LoA (CI 95\%) } \\
\text { (LoA Inf-LoA Sup) }\end{array}$ & $\begin{array}{c}\text { VN } \\
\text { (VN Inf-VN Sup) }\end{array}$ \\
\hline Walking Points & 0.02 & 0.6 & 0.06 & 2.88 & $0.14(-2.74-3.03)$ & $4.0 \pm 0.19(3.80-4.20)$ \\
\hline Walking point gap & 0.003 & 0.19 & 0.01 & 1.3 & $0.01(-1.29-1.32)$ & $1.97 \pm 0.01(1.95-1.99)$ \\
\hline Step width & 0.03 & 0.27 & 0.08 & 2.08 & $0.21(-1.87-2.29)$ & $11.1 \pm 0.29(10.8-11.4)$ \\
\hline Walking base & 0.02 & 0.73 & 0.06 & 2.88 & $0.14(-2.74-3.03)$ & $3.20 \pm 0.18(3.02-3.38)$ \\
\hline
\end{tabular}

Abbreviations: SD: standard deviation; CI: confidence interval; ICC: intraclass correlation coefficient; CV: variation interclass; SEM: standard error of the mean; MDC: minimal detectable change; CR: repeatability coefficient LOA: concordance limit; VN: normality values. Confidence interval 95\%.

\section{Discussion}

Inter-session and intra-session reliability analysis seems to be a key point for the gait system control assessment in order to achieve that the obtained differences in posture and gait activity shows true modifications in walking performance, rather than differences in assessment technique. This research shows that the OptoGait system is a confident tool in order to obtain different spatio-temporal gait data, and this gait and posture assessment device can be used to evaluate the different performance for 
clinical activities (such as clinical treatment or orthopedic devices, e.g., insoles) and their modifications along the treatment intervention.

In our research, all the subjects were barefoot. Shoes could modify the studied variables due to different characteristics, such as drop or shore value.

A key goal in this observational study was to obtain a baseline for the four studied variables in order to detect different data caused by pathologies. The developed trials for the participants in this research did not cause fatigue. On the other hand, a greater number of measurements may achieve higher reliability values.

We checked an uninjured population in order to achieve the reliability for the four variables of walking point, walking point gap, step width, and walking base, which are deeply related to the gait measurement variables analyzed by the OptoGait system.

The SEM values for all four datasets in this trial were very low, suggesting that these data are strong enough to use in the clinical assessment of changes before and after interventions like physical therapy, orthotics devices, or therapeutic exercise interventions. In addition, the OptoGait device seems useful for measuring differences between different participant groups. SEM is defined as a quantitative measurement of the errors that could happen when a participant repeats certain tests; in our trial, the obtained intra-session SEM values were very low, showing a strong absolute reliability.

Our results are close to those reported by previous studies $[1-3,9,14]$. The obtained data may help future clinicians to achieve adequate clinical assessments regarding gait and posture modifications that are due to factors beyond the errors associated with the normal variability of measurements between trials.

\section{Conclusions}

This trial has shown that the OptoGait portable photoelectric system is a strong and reliable tool for the clinical measurement of the walking point, walking point gap, step width, and walking base variables, which are deeply related to gait and posture, with an almost perfect reliability. In addition, these results offer an adequate baseline for clinicians and research.

The OptoGait portable photoelectric system quickly provides useful measurements for clinical assessment or sports evaluation in order to prevent pathology, or to achieve a reliable clinical screening.

Author Contributions: Conceptualization, M.E.L.-I.; Data curation, J.C.-L., M.E.L.-I. and M.S.A.; Formal analysis, M.E.L.-I. and C.C.-L.; Investigation, C.C.-L. and M.S.A.; Methodology, J.C.-L., R.B.-d.-B.-V. and C.C.-L.; Supervision, R.B.-d.-B.-V.; Validation, I.C.-H., M.B.-D.P. and D.R.-S.; Visualization, I.C.-H.; Writing-Original draft, J.C.-L., R.B.-d.-B.-V., M.B.-D.P., D.R.-S. and M.S.A. All authors have read and agree to the published version of the manuscript.

Funding: This research received no external funding

Conflicts of Interest: The authors declare no conflict of interest.

\section{References}

1. García-Pinillos, F.; Latorre-Román, P.A.; Ramirez-Campillo, R.; Roche-Seruendo, L.E. Agreement between spatiotemporal parameters from a photoelectric system with different filter settings and high-speed video analysis during running on a treadmill at comfortable velocity. J Biomech. 2019, 93, 213-219. [CrossRef] [PubMed]

2. Healy, A.; Linyard-Tough, K.; Chockalingam, N. Agreement Between the Spatiotemporal Gait Parameters of Healthy Adults From the OptoGait System and a Traditional Three-Dimensional Motion Capture System. J. Biomech. Eng. 2019, 141, 014501. [CrossRef] [PubMed]

3. Engelson, M.A.; Bruns, R.; Nightingale, C.J.; Bardwell, K.M.; Mason, C.A.; Tu, S.; Nelson, L.; Butterfield, S.A. Validation of the OptoGait System for Monitoring Treatment and Recovery of Post-Concussion Athletes. J. Chiropr. Med. 2017, 16, 163-169. [CrossRef] [PubMed]

4. Eastlack, M.E.; Arvidsen, J.; Snyder-Mackler, L.; Danoff, J.V.; McGarvey, C.L. Interrater reliability of videotaped observational gait-analysis assessments. Phys. Ther. 1991, 71, 465-472. [CrossRef] [PubMed] 
5. Krebs, D.E.; Edelstein, J.E.; Fishman, S. Reliability of observational kinematic gait analysis. Phys. Ther. 1985, 65, 1027-1033. [CrossRef] [PubMed]

6. Saleh, M.; Murdoch, G. In defence of gait analysis. Observation and measurement in gait assessment. J. Bone Jt. Surg. Br. Vol. 1985, 67, 237-241. [CrossRef]

7. Montes-Alguacil, J.; Páez-Moguer, J.; Cebrián, A.M.J.; Muñoz, B.A.; Noguerón, G.G.; Morales-Asencio, J.M. The influence of childhood obesity on spatio-temporal gait parameters. Gait Posture 2019, 71, 69-73. [CrossRef] [PubMed]

8. Maynard, V.; Bakheit, A.M.; Oldham, J.; Freeman, J. Intra-rater and inter-rater reliability of gait measurements with CODA mpx30 motion analysis system. Gait Posture 2003, 17, 59-67. [CrossRef]

9. Lee, M.M.; Song, C.H.; Lee, K.J.; Jung, S.W.; Shin, D.C.; Shin, S.H. Concurrent validity and test-retest reliability of the OptoGait photoelectric cell system for the assessment of spatio-temporal parameters of the gait of young adults. J. Phys. Ther. Sci. 2014, 26, 81-85. [CrossRef] [PubMed]

10. Lienhard, K.; Schneider, D.; Maffiuletti, N.A. Validity of the Optogait photoelectric system for the assessment of spatial-temporal gait parameters. Med. Eng. Phys. 2013, 35, 500-504. [CrossRef]

11. Webster, K.E.; Wittwer, J.E.; Feller, J.A. Validity of the GAITRite walkway system for the measurement of averaged and individual step parameters of gait. Gait Posture 2005, 22, 317-321. [CrossRef]

12. Besser, M.P.; Kmieczak, K.; Schwartz, L.; Snyderman, M.; Wasko, J.; Silverstein, L.S. Representation of temporal spatial gait parameters using means in adults without impairment. Gait Posture 1999, 9, 113.

13. Landis, J.R.; Koch, G.G. The measurement of observer agreement for categorical data. Biometrics 1977, 33, 159-174. [CrossRef] [PubMed]

14. Bernal, A.G.; Becerro-de-Bengoa-Vallejo, R.; Losa-Iglesias, M.E. Reliability of the OptoGait portable photoelectric cell system for the quantification of spatial-temporal parameters of gait in young adults. Gait Posture 2016, 50, 196-200. [CrossRef] [PubMed]

(C) 2020 by the authors. Licensee MDPI, Basel, Switzerland. This article is an open access article distributed under the terms and conditions of the Creative Commons Attribution (CC BY) license (http://creativecommons.org/licenses/by/4.0/). 